This item was submitted to Loughborough's Research Repository by the author.

Items in Figshare are protected by copyright, with all rights reserved, unless otherwise indicated.

\title{
Hair density distribution profile to evaluate yarn hairiness and its application to fabric simulations
}

PLEASE CITE THE PUBLISHED VERSION

http://dx.doi.org/10.1080/00405000701462419

\section{PUBLISHER}

(C) Textile Institute. Published by Taylor and Francis.

\section{VERSION}

AM (Accepted Manuscript)

\section{PUBLISHER STATEMENT}

This work is made available according to the conditions of the Creative Commons Attribution-NonCommercialNoDerivatives 4.0 International (CC BY-NC-ND 4.0) licence. Full details of this licence are available at: https://creativecommons.org/licenses/by-nc-nd/4.0/

\section{LICENCE}

CC BY-NC-ND 4.0

\section{REPOSITORY RECORD}

Ozkaya, Yasar A., Memis Acar, and Michael R. Jackson. 2019. "Hair Density Distribution Profile to Evaluate Yarn Hairiness and Its Application to Fabric Simulations". figshare. https://hdl.handle.net/2134/19367. 


\title{
HAIR DENSITY DISTRIBUTION PROFILE TO EVALUATE YARN HAIRINESS AND ITS APPLICATION TO FABRIC SIMULATIONS
}

\author{
Yasar A. Ozkaya, Memis Acar and Mike R. Jackson \\ Loughborough University, School of Mechanical and Manufacturing Engineering, \\ Leicestershire LE113TU, UK \\ Corresponding author: m.acar@lboro.ac.uk
}

\begin{abstract}
This paper describes a new method for yarn hairiness evaluation that calculates the hair densities at varying distances from the yarn core using an image processing technique. The method is based on integrating the number of pixels for distances incremented by a pixel size from the yarn core edge. The experiments with various yarns showed that the hair density distribution profile (HDDP) exhibits two different exponential behaviors one below and the other above approximately $0.75 \mathrm{~mm}$ from the core. A total hairiness index (THw) is also calculated using the total number of hair pixels. A good correlation is observed between the proposed THw and Uster's H index especially for grey cotton and cotton/polyester samples. A novel technique that generates realistic yarn simulations using the total hairiness and the hairiness distribution data along with the diametric irregularity is introduced. The simulations created are compared with actual yarn images in a qualitative manner. A simple single jersey knitted fabric simulation algorithm is also described utilizing the yarn simulation including hair distribution data, which gives a more realistic simulated fabric appearance.
\end{abstract}

Keywords: Yarn hairiness, hair-density distribution, yarn simulation, fabric simulation

\section{Introduction}

Since its first introduction in 1950s, many methods and indices have been developed to assess the hairiness characteristic of yarns. However only two methods are commonly accepted by industry today, which are the so called 'total hairiness index' of Uster Tester and the measurement of number of hairs at certain distances from the core as in Zweigle G566 and Shirley YHM. Nevertheless, hairiness measurement still appears to be far from being standardized, due to the lack of a universally accepted hairiness index and the fact that the indices for hairiness don't have a clear correlation with each other since they describe different characteristics (Barella et al-1992). Furthermore, the literature recognizes some shortcomings of both indices such as the lack of hair length information of the total hairiness index of Uster Tester and the sensor resolution limits of photo-sensor based testers, such as Zweigle Hairiness Tester that result in miscounting especially at close vicinity of the core. The latter has been investigated in detail by the authors, where it was shown that at especially 1 $\mathrm{mm}$ distance from the core, the photo sensor resolution of commercial testers based on counting the number of hairs would be inadequate (Ozkaya et al, 2005b). Notwithstanding this, for yarns possessing mainly short-hair hairiness such as rotor spun yarns, this technique gives lower hairiness values since it is not possible to detect hairs standing at less than $1 \mathrm{~mm}$ from the core using the photo sensor method (Barella et.al. 1992). Describing the hairiness by 
a single 'total hairiness' index also has significant shortcomings. A report by Suessen (Spinnovation, 3/2002) indicates that short hairs contribute to a good textile touch in the fabric while fiber ends exceeding $2 \mathrm{~mm}$ are generally found to disturb the subsequent processing steps causing high additional costs and affecting the performance of the finished textile article. They state that the measuring method according to USTER Hairiness $(\mathrm{H})$ does not by far reflect the actual yarn conditions since no difference is made between long and short hairs. In contrast, Zweigle's measuring method classifies the yarns according to their hair length only. They showed that the hairiness measurements of yarn samples produced by different spinning machines and with different quality characteristics associated with their hairiness, were significantly different in terms of Zweigle S3 index while only slightly different in terms of Uster's $\mathrm{H}$ index.

A new concept called 'Hair Density Distribution Profile (HDDP)' that aims to simultaneously give not only a measure of total hairiness (similar to Uster Tester) but also the distribution of the hairs at certain distances from the core (similar to Zweigle G566) is explained in this paper.

\section{Description of Technique}

A CCD line-scan system was developed at to capture digital image of the yarns. A Dalsa Spark 2048-pixel line scan camera with a $100 \mathrm{~mm}$ macro lens giving 1x magnification is used for image acquisition. The maximum line rate of the camera is $18.8 \mathrm{kHz}$. The images are transferred to a PC through a Viper Digital frame grabber. The pixel size of the camera is 14 $\mu \mathrm{mx} 14 \mu \mathrm{m}$ with a fill factor of $100 \%$. The yarns are backlit using a $50 \mathrm{~W}$ tungsten filament bulb. The line acquisition is triggered using an optical encoder attached to the yarn transfer system.

The line scan camera allows a scanning resolution of $14 \mathrm{scans} / \mathrm{mm}$ at a yarn speed of $80 \mathrm{~m} / \mathrm{min}$. The scanning resolution can be increased by decreasing the yarn transfer speed. However, 14 scans/mm is found to be the lower limit for yarn scanning since the hairs are lost below this resolution giving lower hairiness values. Images are processed using Wit 7.1 image processing software and $\mathrm{C}$ programming language.

The image processing algorithm for separation of hairs, core and the background for backlit yarn images is described by Ozkaya et.al. (2005a). Figure 1 shows the images of an Ne 10, $65 / 35$ polyester/cotton yarn scanned at 70 scans/mm before and after image processing.

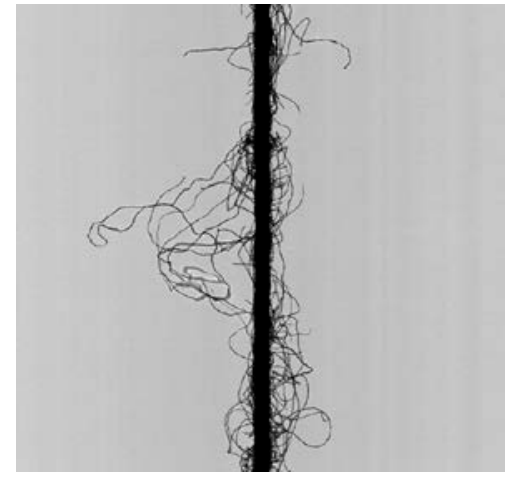

(a)

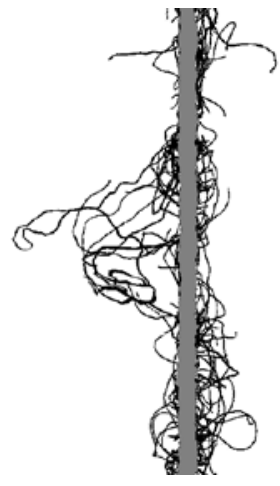

(b)

Figure 1 Raw (a) and processed (b) yarn images 
A series of tests were carried out using 22 ring spun yarn samples shown in Table 1 . For each yarn, 1250 images of $2 \mathrm{~cm}$ long segments corresponding to a total of $25 \mathrm{~m}$ yarn length were stored in the computer for the analysis. This length was found adequate following the observation of change in hairiness and hairiness variation for increasing test lengths which converged after a test length of around $10 \mathrm{~m}$ for all samples (Ozkaya et al 2005b). For real time storing of the images, the test speed was kept low at $5 \mathrm{~m} / \mathrm{min}$.

Table 1 Yarn samples used in the experiments

\begin{tabular}{|c|c|c|c|}
\hline Sample \# & Blend & Yarn Count & $\begin{array}{c}\text { Twist } \\
\text { turns/inch) }\end{array}$ \\
\hline 1 & 65/35 Cotton/Polyester & Ne 30/1 & 21.59 \\
\hline 2 & $100 \%$ Cotton & Ne 10/1 & 12.33 \\
\hline 3 & $100 \%$ Cotton & Ne 10/1 & 10.43 \\
\hline 4 & $65 / 35$ Cotton/Polyester & Ne 30/1 & 19.8 \\
\hline 5 & 65/35 Polyester/Cotton & Ne 20/1 & 16.1 \\
\hline 6 & $65 / 35$ Polyester/Cotton & Ne 10/1 & 11.38 \\
\hline 7 & 65/35 Cotton/Polyester & Ne 10/1 & 12.33 \\
\hline 8 & $100 \%$ Cotton & Ne 10/1 & 11.38 \\
\hline 9 & $65 / 35$ Polyester/Cotton & Ne 10/1 & 12.3 \\
\hline 10 & $60 / 40$ Acrylic/Wool & Nm 30/1 & 12.2 \\
\hline 11 & $60 / 40$ Acrylic/Wool & Nm 30/1 & 9.65 \\
\hline 12 & $70 / 30$ Acrylic/Wool & Nm 32/1 & 13.08 \\
\hline 13 & $100 \%$ Cotton & Ne 60/1 & 29.21 \\
\hline 14 & $100 \%$ Cotton & Ne 10/1 & 11.05 \\
\hline 15 & $100 \%$ Cotton & Ne 20/1 & 15.24 \\
\hline 16 & $100 \%$ Cotton & Ne 30/1 & 19.56 \\
\hline 17 & $100 \%$ Cotton & Ne 30/1 & 22.36 \\
\hline 18 & $100 \%$ Cotton & Ne 30/1 & 21.96 \\
\hline 19 & $100 \%$ Cotton & Ne 18/1 & - \\
\hline 20 & $100 \%$ Cotton & Ne 12/1 & - \\
\hline 21 & $100 \%$ Cotton & Ne 30/1 & - \\
\hline 22 & $100 \%$ Cotton & Ne 28/1 & - \\
\hline
\end{tabular}

\section{Hair Density Distribution Profile}

The hair density distribution is obtained by counting the number of pixels recording hairs at every distance incremented by 14 microns (the pixel size) from the core edge. The ratio of number of pixels covered by hairs to the total number of pixels tracked for each distance gives the probability of the existence of a hair at that distance.

USTER $\mathrm{H}$ hairiness indices were measured for all yarns listed in Table 1. Each result presented is the average of three $100-\mathrm{m}$ specimens, tested at $400 \mathrm{~m} / \mathrm{min}$ test speed. Then a total hairiness index (THw) is calculated from the total area under the HDDP by multiplying the corresponding average number of hair pixels along one metre yarn length by the area of one pixel in $\mathrm{mm}^{2}$. The hairiness variation is also calculated from the total hairiness values of successive yarn segments. The length of the sections is selected as $10 \mathrm{~mm}$ in order to compare the THw CV\% with the hairiness variation of Uster Tester, however, the software also provides the variation values for 20 different lengths varying from $1 \mathrm{~mm}$ to $2500 \mathrm{~mm}$ in order to calculate the CV-Length curves for hairiness. 


\section{Yarn simulations using HDDP}

In order to demonstrate one of the possible applications of HDDP, a yarn simulation algorithm is developed. The basic principle is to generate the yarn appearance in vector form and then to convert this vector image into an 8-bit bitmap for a given resolution. The diameter and total hairiness data for 1 and $10 \mathrm{~mm}$ sampling lengths respectively are used along with the HDDP to generate the simulations.

A further algorithm is developed to add the hairiness effect on yarn and fabric simulations that uses the total hairiness (THw) values from $1 \mathrm{~cm}$ yarn segments and the Hair Density Distribution Profile (HDDP). The first task is to map the HDDP according to the resolution of simulation as shown in Figure 2 where the HDDP that originally has around 1815 DPI resolution is mapped onto 127 DPI resolution by taking the average of every successive 14.3 data points in the original profile.

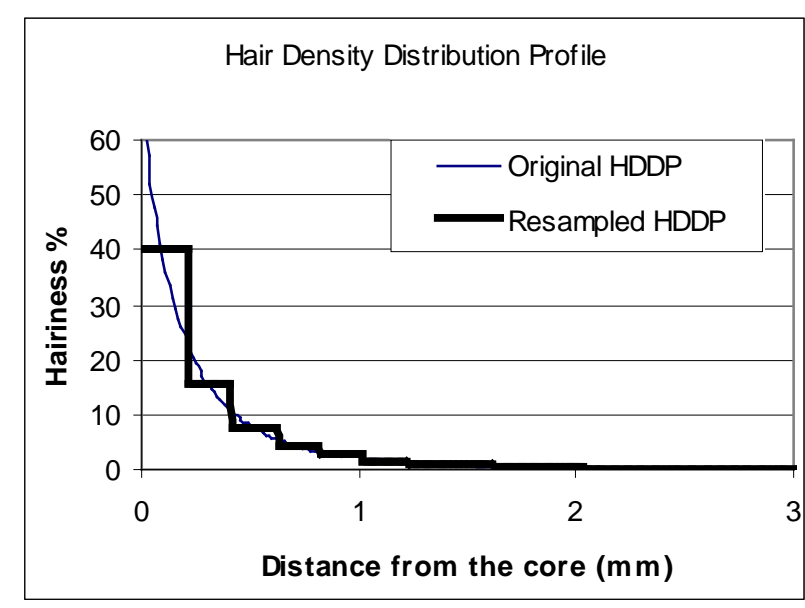

Figure 2 The original HDDP mapped for 127DPI resolution

The next task is to distribute the THw values for $1 \mathrm{~cm}$ yarn segments around the yarn core according to HDDP. An iterative algorithm selects points at random distances from the core for randomly selected $1 \mathrm{~cm}$ core sections and adds random intensity values to these points, where the intensities correspond to given hair areas. Two basic rules are applied: (i) the total intensity assigned for a given $1 \mathrm{~cm}$ yarn segment cannot exceed the corresponding THw for that segment, (ii) the integration of intensities for all core portions for a certain distance from the core cannot exceed the probability that a hair exists at that distance, which is found from the HDDP. A queue type data structure is used in the algorithm that throws away the $1 \mathrm{~cm}$ yarn segments for which the THw values are reached and continues random selection of yarn segments among the remaining ones. Furthermore, if the amount of hairs plotted at a given distance from the core reaches the maximum amount determined by HDDP then again, that distance is no longer selected in the random hair distribution process. The algorithm continues until all the THw values are allocated to yarn segments.

Once the hair-distance distributions are calculated for all yarn segments, the final task is to spread hairs within $1 \mathrm{~cm}$ long yarn segment within the simulation. To do this, the number of pixels that corresponds to a $1 \mathrm{~cm}$ segment in the simulation is first calculated and then for each distance from the core, the total hairiness for that segment is randomly distributed over these pixels with random intensities, where the intensities of the pixels again correspond to varying hair areas. 


\section{Knitted fabric simulation using HDDP}

A single jersey knitted fabric simulation algorithm is developed that uses the simulated yarn based on the above principle. A simplified 2D version of the loop geometry studied by Suh [1967] is used. Figure 3 shows the plan view of jersey loops and the loop construction used in this model. Here, $\mathrm{P}$ and $\mathrm{W}$ are the course and wale spacings.

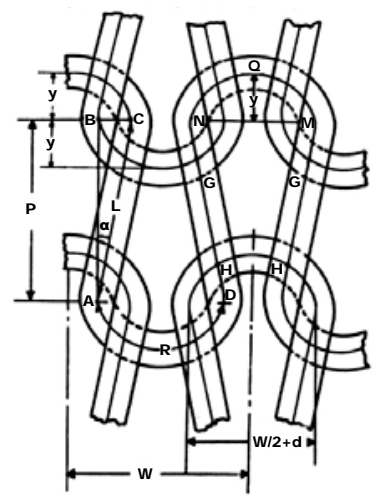

(a)

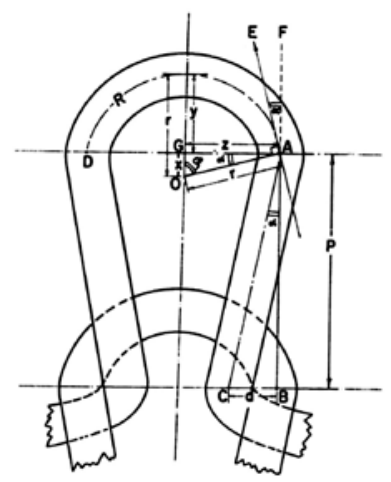

(b)

Figure 3 Loop geometry. a) Top view of jersey loops; b) Construction of sinker and needle loops (Suh [1967], p419)

The arc DA in Figure 3 is assumed to be a semicircle centered at $\mathrm{O}$ and the curvature length $\mathrm{R}$ can be found using the following equation;

$$
R=2 . r . \phi
$$

Where $\mathrm{r}$ can be found using,

$$
r=\frac{(W+2 \cdot P \cdot \cos (\phi))}{4\left[\cos ^{2}(\phi)+2 \cdot \sin (\phi)\right]}
$$

The length of the loop legs $\mathrm{L}(\mathrm{AC})$ is calculated from the following equation,

$$
L=P-r \cdot \cos (\phi)
$$

Finally, the stitch length (S) of a loop is found as follows;

$$
S=2 .(L+R)
$$

It should be noted that the course curvature depicted in Figure 4 is not taken into account when calculating the leg length $\mathrm{L}$; therefore the actual value of $\mathrm{L}$ would be longer than that is found from equation 3. However, since the simulations are generated using 2D geometry, the legs would appear to have the length calculated from equation 3. Nevertheless, the main problem of using 2D geometry is the miscalculation of the stitch length $\mathrm{S}$. For example, if the stitch length were calculated as $6 \mathrm{~mm}$ while it was say $7 \mathrm{~mm}$; the $100^{\text {th }}$ loop for instance, would correspond to the yarn section at $600 \mathrm{~mm}$ rather than $700 \mathrm{~mm}$. As a solution to this problem, the lengths $\mathrm{L}$ and $\mathrm{R}$ are calculated using 2D geometry; however the stitch length $\mathrm{S}$ can be entered manually in the program to enable a more accurate simulation. The most accurate way to calculate the stitch length of a fabric is to unravel certain amount of loops from the knitted fabric and then divide the total length of the unraveled yarn to the corresponding number of loops.

The leg angle $\varphi$ is taken as $75^{\circ}$ by default, however the program can also calculate it automatically for a given stitch length (S) and course and wale densities by solving equations 1-4 for $\varphi$. 


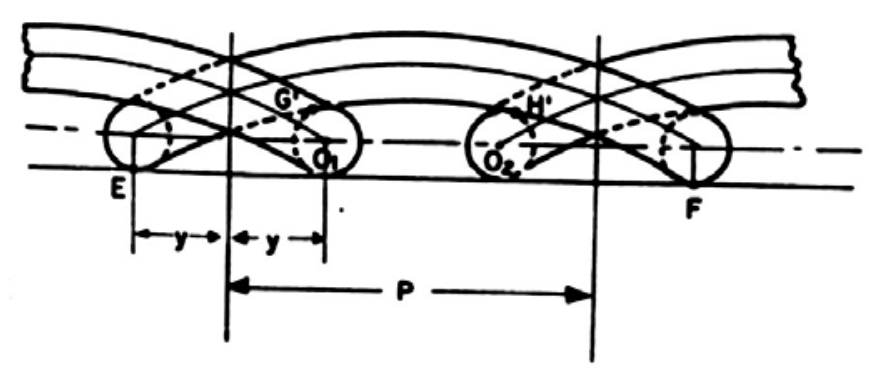

Figure 4 Side view of jersey loops (Suh [1967], p419)

As in the yarn simulation, the knitted fabric simulation is first calculated in vector form and then digitized by calculating the areas of overlap between pixels and the yarns and assigning grey level values to the pixels proportional to these areas as depicted in Figure 5. The legs are defined as rectangles with a length of L lying at a degree of $\varphi$ to the horizontal axis. The width of the rectangle is calculated from the average yarn diameter corresponding to that particular segment. The curvatures are defined in a similar fashion as semicircles with a curvature length of $\mathrm{R}$ and a thickness corresponding to the average diameter of that section.

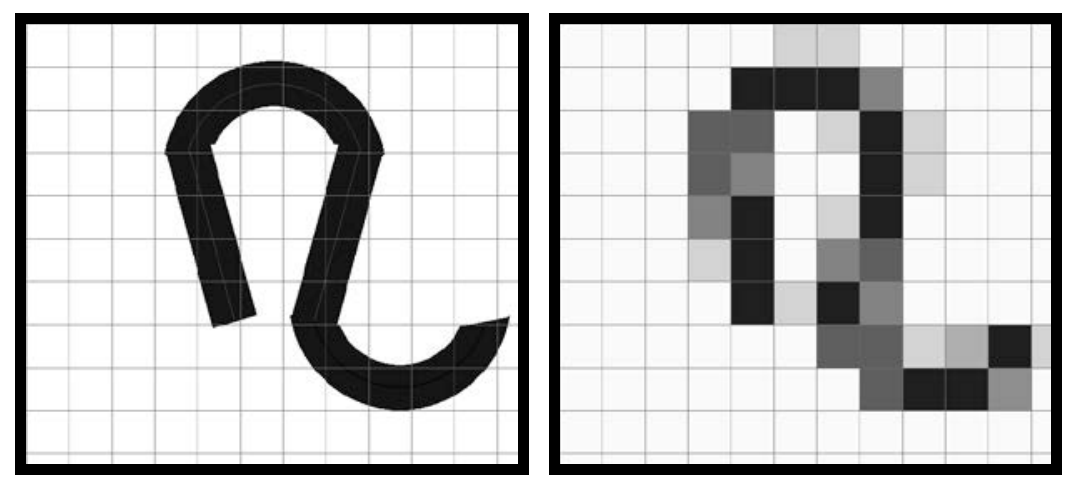

Figure 5 Digitization of geometrically defined loops

In order to add the hairiness effect to the knitting simulations, the hairiness distribution is calculated according to the THw values of $1 \mathrm{~cm}$ segments and the HDDP using the iterative algorithm for yarn board simulation. The main difference from the yarn board simulation is that when plotting the hairs according to their distances from the core, the positions of the pixels are calculated perpendicular to the yarn segment, which can be leg or the curvature of the loop.

\section{Results and Discussion}

Figure 6a shows a typical hair-density profile calculated for a test length of $25 \mathrm{~m}$. On a logarithmic scale, two different linear trends are observed as can be seen in Figure 6b. These trends are found to be separated at around $0.75 \mathrm{~mm}$ from the core for all samples. Table 2 shows the slopes of the best-fit lines fitted to the HDDP on logarithmic scale below and above $0.75 \mathrm{~mm}$ from the core, along with the intercept and $\mathrm{R}^{2}$ values. It can be seen that the trend lines are quite significant with $\mathrm{R}^{2}$ values of higher then 0.98 and the slopes are clearly different for values below and above $0.75 \mathrm{~mm}$. Following this, the part of HDDP below 0.75 $\mathrm{mm}$ is considered to represent the short hair hairiness and the rest the long hair hairiness, which have different statistical characteristics. 


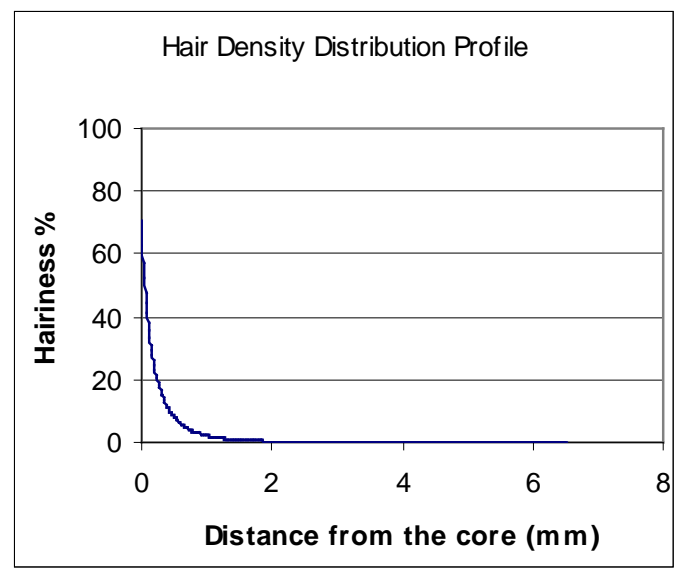

(a)

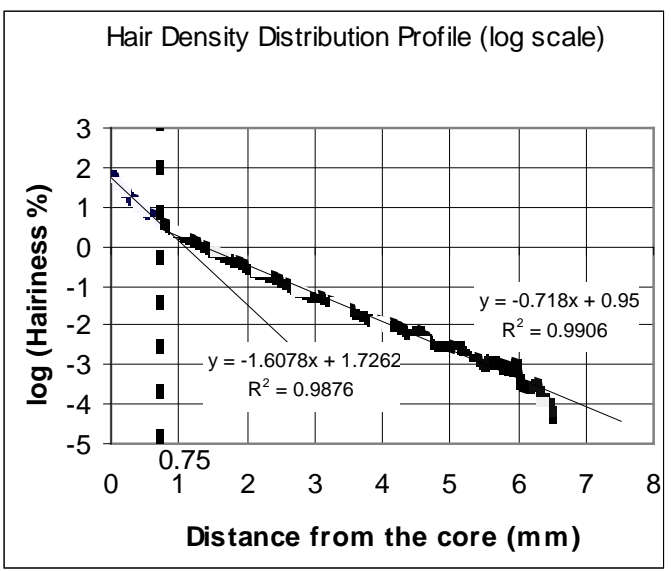

(b)

Figure 6 Hair Density Distribution Profile (HDDP) of a typical yarn on normal (a) and logarithmic (b) scales

Table 2 Slopes (m), offsets (b) and R2 values of the best-fit lines to the HDDP on logarithmic scale for measurements below and above $0,75 \mathrm{~mm}$ from the core $(y=m x+b)$.

\begin{tabular}{|c|c|c|c|c|c|c|}
\hline \multirow[t]{2}{*}{ Sample\# } & \multicolumn{3}{|c|}{$\mathrm{L} \leq 0.75 \mathrm{~mm}$} & \multicolumn{3}{|c|}{$\mathrm{L}>0.75 \mathrm{~mm}$} \\
\hline & $\mathrm{m}$ & $\mathrm{b}$ & $\mathrm{R}^{2}$ & $\mathrm{~m}$ & $\mathrm{~b}$ & $\mathrm{R}^{2}$ \\
\hline 1 & -1.67501 & 1.409163 & 0.984204 & -0.68557 & 0.526406 & 0.993075 \\
\hline 2 & -1.58001 & 1.650767 & 0.990255 & -0.63263 & 0.776662 & 0.991058 \\
\hline 3 & -1.51647 & 1.681013 & 0.990979 & -0.67315 & 0.909738 & 0.993908 \\
\hline 4 & -1.66757 & 1.460766 & 0.987424 & -0.68911 & 0.542514 & 0.985387 \\
\hline 5 & -1.64486 & 1.518171 & 0.990141 & -0.75078 & 0.675394 & 0.990886 \\
\hline 6 & -1.58019 & 1.465919 & 0.992387 & -0.71122 & 0.616343 & 0.985147 \\
\hline 7 & -1.44227 & 1.638258 & 0.988719 & -0.59056 & 0.842201 & 0.99238 \\
\hline 8 & -1.50156 & 1.571486 & 0.990156 & -0.6195 & 0.728215 & 0.987933 \\
\hline 9 & -1.558 & 1.396813 & 0.985396 & -0.60129 & 0.480479 & 0.980133 \\
\hline 10 & -1.32814 & 1.220939 & 0.979529 & -0.42969 & 0.411879 & 0.988402 \\
\hline 11 & -1.34831 & 1.397208 & 0.976136 & -0.42968 & 0.584741 & 0.989934 \\
\hline 12 & -1.5059 & 1.678069 & 0.979368 & -0.43437 & 0.734776 & 0.98675 \\
\hline 13 & -1.56168 & 1.21188 & 0.975801 & -0.54418 & 0.294518 & 0.986958 \\
\hline 14 & -1.42761 & 1.659269 & 0.989584 & -0.54796 & 0.83444 & 0.989744 \\
\hline 15 & -1.38377 & 1.55237 & 0.985808 & -0.51648 & 0.762061 & 0.992284 \\
\hline 16 & -1.61861 & 1.483354 & 0.985894 & -0.596 & 0.547817 & 0.990131 \\
\hline 17 & -1.46676 & 1.50621 & 0.984425 & -0.58938 & 0.725749 & 0.994914 \\
\hline 18 & -1.46485 & 1.516771 & 0.985495 & -0.60036 & 0.758218 & 0.996364 \\
\hline 19 & -1.6878 & 1.495208 & 0.986289 & -0.64342 & 0.500104 & 0.985446 \\
\hline 20 & -1.37134 & 1.644449 & 0.990237 & -0.572 & 0.884903 & 0.99146 \\
\hline 21 & -1.49901 & 1.464517 & 0.98711 & -0.61066 & 0.626112 & 0.987756 \\
\hline 22 & -1.43648 & 1.362259 & 0.978574 & -0.56858 & 0.602718 & 0.990985 \\
\hline
\end{tabular}

Table 3 shows the THw and USTER $\mathrm{H}$ indices test results. Figure 7 shows the comparison of THw calculated from backlit images with USTER's H index. A good correlation has been observed between the two indices with a correlation coefficient (R) of 0.93; however there is a small offset where USTER $\mathrm{H}$ index has a non-zero value 
where THw is zero. One of the main reasons for this is the difference between the definition of core boundaries in backlit illumination used in THw measurement and dark field illumination to measure USTER $\mathrm{H}$, where there is a more significant contribution of surface fibers to total hairiness in dark-field illuminated images.

Table 3 Yarn samples used in the experiments

\begin{tabular}{|c|c|c|c|c|}
\hline Sample \# & THw & USTER H & THw CV\% & USTER H CV\% \\
\hline 1 & 1.92 & 6.41 & 25.16 & 24.65 \\
\hline 2 & 3.23 & 10.04 & 21.06 & 22.41 \\
\hline 3 & 3.57 & 11.93 & 20.84 & 22.72 \\
\hline 4 & 2.35 & 7.1 & 25.46 & 24.79 \\
\hline 5 & 2.37 & 7.72 & 22.07 & 23.70 \\
\hline 6 & 2.88 & 9.29 & 20.32 & 21.20 \\
\hline 7 & 2.72 & 9.19 & 21.1 & 20.57 \\
\hline 8 & 3.22 & 10.48 & 21.19 & 22.71 \\
\hline 9 & 2.67 & 8.88 & 20.27 & 20.83 \\
\hline 10 & 1.86 & 6.9 & 38.23 & 33.04 \\
\hline 11 & 2.63 & 10.37 & 30.59 & 27.48 \\
\hline 12 & 3.93 & 13.48 & 34.44 & 37.83 \\
\hline 13 & 1.51 & 4.06 & 27.8 & 25.12 \\
\hline 14 & 3.47 & 12.04 & 24.28 & 25.75 \\
\hline 15 & 2.74 & 9.57 & 24.13 & 24.56 \\
\hline 16 & 1.86 & 6.44 & 25.84 & 29.19 \\
\hline 17 & 2.12 & 8.33 & 23.83 & 24.85 \\
\hline 18 & 2.05 & 8.37 & 23.27 & 25.69 \\
\hline 19 & 1.83 & 8.19 & 29.14 & 30.04 \\
\hline 20 & 3.81 & 11.29 & 24.26 & 27.55 \\
\hline 21 & 1.97 & 6.64 & 21.32 & 24.25 \\
\hline 22 & 2.46 & 8.02 & 24.35 & 28.43 \\
\hline
\end{tabular}

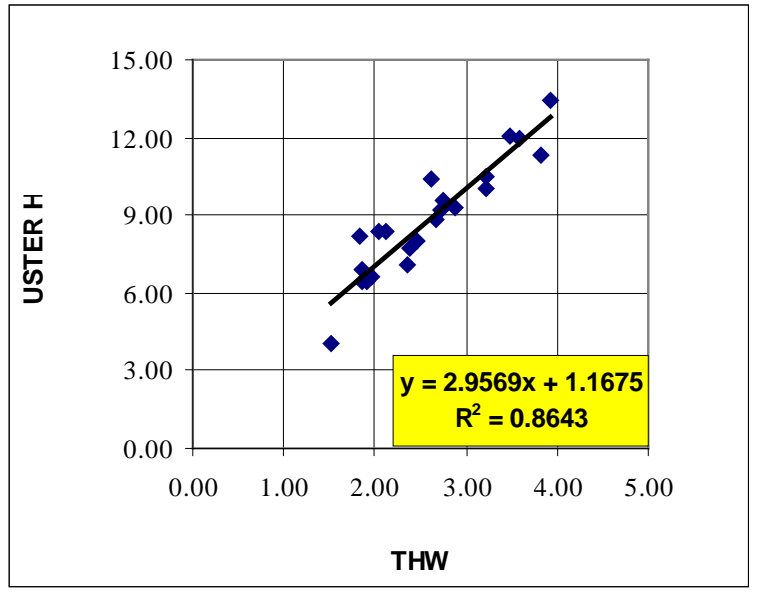

(a)

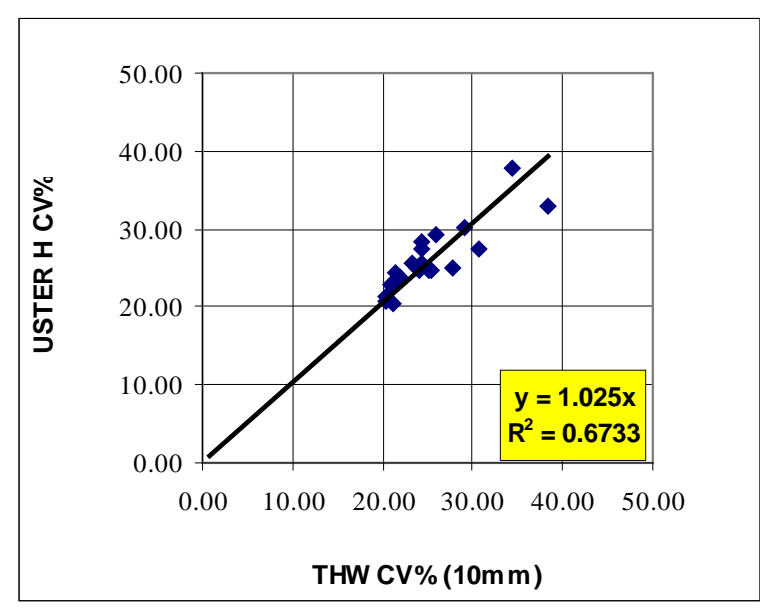

(b)

Figure 7 Comparison of backlit THw (a) and THw CV\% (b) with USTER H index and USTER H CV\% 
The CV\% values calculated from THw values for $1 \mathrm{~cm}$ sampling length exhibited a reasonable agreement with USTER H CV\% values as shown in the Figure b. The measurements from two testers were of the same order of value.

The correlation coefficient was higher at around 0.97 for grey cotton-cotton/polyester samples only (excluding samples 10-12, which are dyed wool/acrylic blends and samples 16-18, which are dyed cotton). This suggests that different blends and colours may have an adverse influence on the correlation between the two indices since material and colour affect the amount of light scattered from the protruding hairs in the USTER Tester. All excluded samples fell above the trend line shown in Figure 7a, which means that with reference to the relation between USTER $\mathrm{H}$ and THw for grey cotton-cotton/polyester yarns, USTER $\mathrm{H}$ exhibited higher hairiness for these samples.

Figure 8a and b show the actual picture of a yarn wound onto a 2"x2" board with $1 \mathrm{~mm}$ spacing and the simulation using the diameter data only acquired from the developed system respectively. It is clear that the hairiness has a very significant effect on the appearance of the yarn and the diameter data alone, although contains diametric variation, is not adequate for yarn simulation.

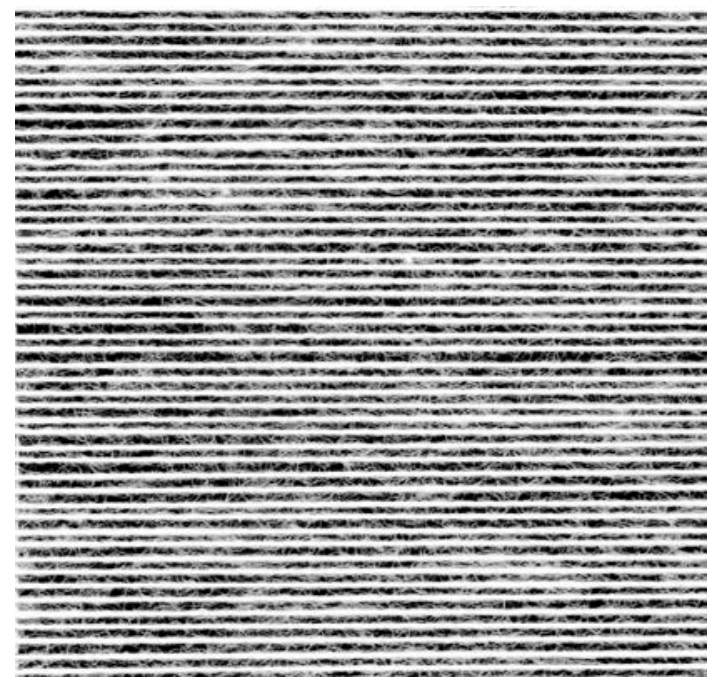

(a)

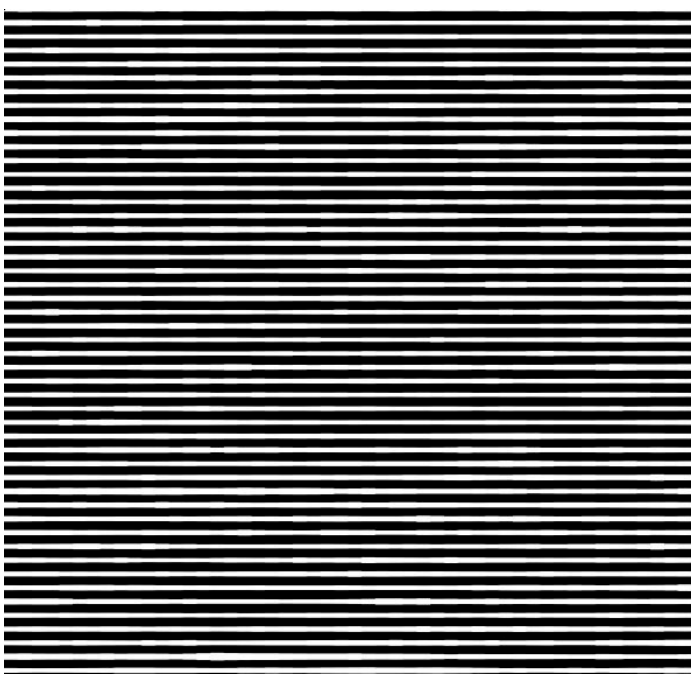

(b)

\section{Figure 8 a) The actual picture of a yarn bundle; b) The simulation using the diameter data}

Figure 9 shows (a) the actual picture of the yarn wound on a 2"x2" yarn board and (b) the yarn board simulation generated using the combined diameter and hairiness data. It is clear that the simulation is much more realistic in this case than the simulation generated using the diameter data alone. 


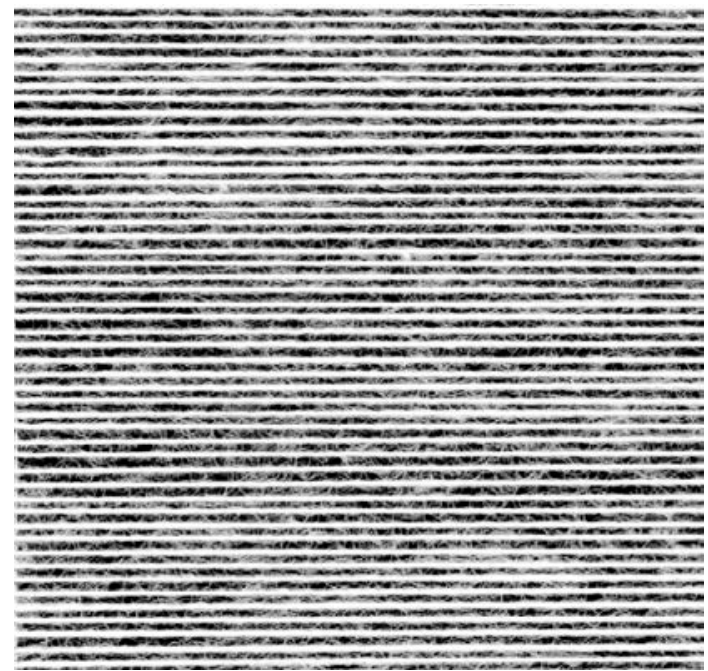

(a)

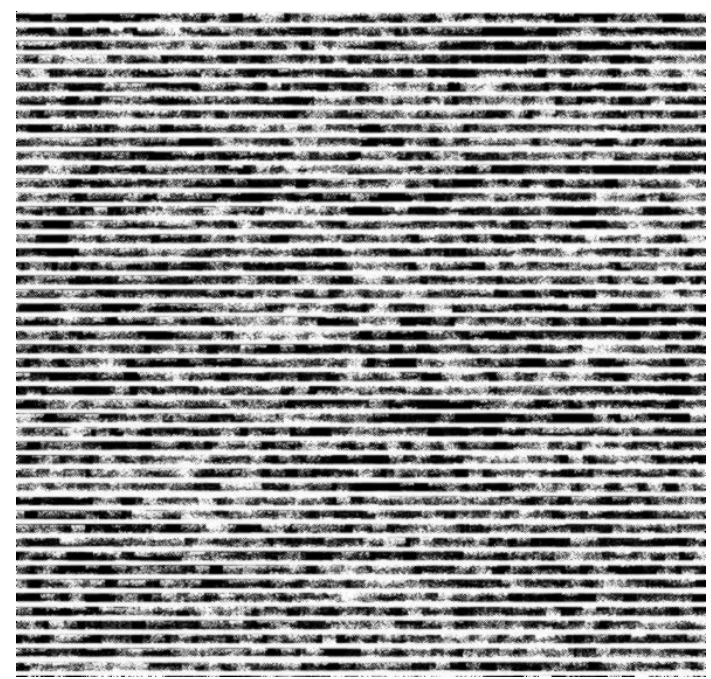

(b)

Figure 9 a) The actual picture of the yarn sample, b) Corresponding yarn board simulation using the combined hairiness and diameter data.

Figure 10 shows knitting simulations using (a) diameter data only and (b) diameter and hairiness data together for yarn sample 12. Here, the course and wale densities are taken as 18 and 15 loops per inch respectively and the knitting machine is assumed to have 220 needles. It can clearly be seen that the effect of yarn irregularity and the hairiness on the simulated knitted fabric is to produce a more realistic fabric simulation.

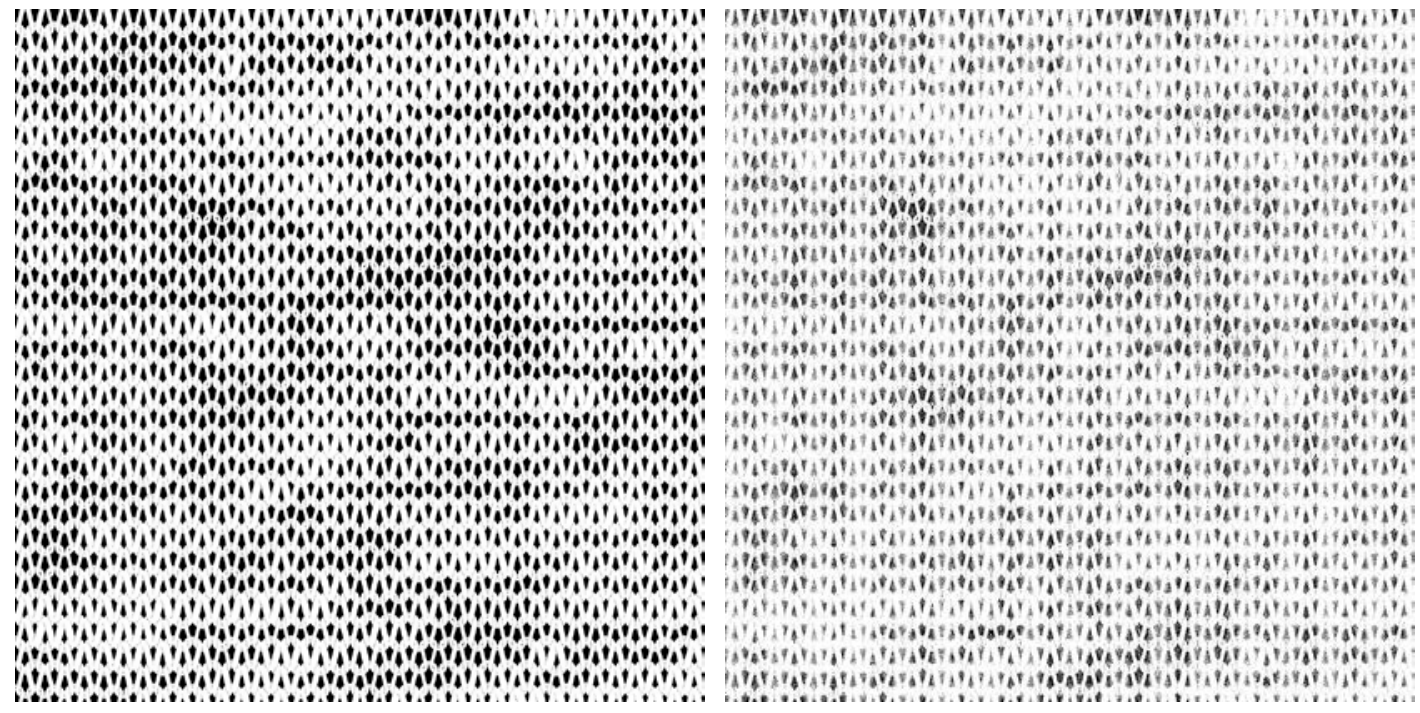

(a)

(b)

Figure 10. Knitting simulation (18 courses/inch, 15 wales/inch, 220 needles). a) With diameter data; b) With hairiness and diameter data 


\section{Conclusions}

A new method of defining yarn hairiness that takes into account both the number of hairs and hair lengths, referred to as Hair Density Distribution Profile (HDDP) is introduced, which gives the probabilistic distribution of hairs around the yarn core with 14 micron accuracy. Two different trends are observed in the profiles on logarithmic scale splitting at around 0.75 $\mathrm{mm}$. The THw index found from the total number of hair pixels in the images agreed very well with Uster Tester's $\mathrm{H}$ index for grey cotton-cotton/polyester samples. But, colour or different blends appeared to decrease this correlation. This is believed to be due to the fact that colour and material can affect the measurements on Uster Tester since they scatter the light differently while such yarn characteristics have no effect on backlit images used in determining HDDP. A novel yarn and fabric simulation principle based on using the actual diameter and hairiness data together is described. The hairiness has a clear affect on the appearance of fabrics and this technique is believed to be superior to the existing simulation systems available in the market. Based on HDDP, a dynamic model can be developed in the future to estimate the change in density distribution when the yarn is woven or knitted and after the finishing processes in order to predict fabric properties that are affected by hairiness such as the appearance and handle.

\section{Acknowledgements}

The financial support of the Wolfson School of Mechanical and Manufacturing Engineering, Loughborough University, UK, is gratefully acknowledged.

\section{References}

Barella, A., Coll-Totosa, L., Espiell, G. S., \& Bardi, X. (1992). Neue Gesichtspunkte bei der Messung und Bewertung der Haarigkeit [New criteria for the measurement and evaluation of hairiness]. Textil Praxis, 47 (12), 1116-1125.

Spindelfabrik Suessen, (2002) "Three Years of Practical Experience with the EliTe Q CompactSet in Short-Staple Spinning” Spinnovation, No.17 -March 2002

Suh, M., (1967) A Study of the Shrinkage of Plain Knitted Cotton Fabric, Based on the Structural Changes of the Loop Geometry Due to Yarn Swelling and Deswelling. Text. Res. J., 37, 417-431

Ozkaya, Y.A., Acar, M. and Jackson, M.R., (2005a) "Digital Image Processing And Illumination Techniques For Yarn Characterization”, J of Electronic Imaging, 14(2), 2005, 13 pp.

Ozkaya, Y.A., Acar, M. and Jackson, M.R., (2005b) "Simulation of Photo-Sensor Based Hairiness Measurement Using Digital Image Analysis”, Journal of the Textile Institute (Submitted). 